\title{
Myxedema Coma: a Review
}

Talha Abderrahim, Bkiyar Houssam, and Housni Brahim

\begin{abstract}
Myxedema coma, represents the extreme degree of severity of hypothyroidism, whose mortality can reach very high percentages, therefore, it is a true medical emergency. In general, its development is associated with the presence of a triggering factor in a controlled hypothyroid patient and manifests with multisystem alteration. Currently, tools have been developed for clinical diagnosis that use the profile and clinical models, and have good sensitivity-specificity. They allow an early diagnosis which favored the early start of treatment and therefore improves the prognosis. The patient with myxedema coma needs an integral approach, with intensive treatment and close monitoring of hemodynamic parameters. However, the basis of treatment remains hormone replacement, which should be initiated with a combination of levothyroxine and triiodothyronine.
\end{abstract}

Index Terms - Myxedema Coma; Hypothyroidism; Intensive care

\section{INTRODUCTION}

This entity was described for the first time at the beginning of 1900, as the result of severe and long-term untreated hypothyroidism (HT). The term myxedema coma (MC), refers to a state that represents the extreme degree of severity HT, whose mortality can be very high, therefore, it is a true medical emergency, which deserves to be diagnosed and managed quickly and intensively. Fortunately, today it is not a frequent entity. An approximate incidence of 0.22 cases per million inhabitants/year is estimated [1], [2]. Currently, clinical tools have been developed for its diagnosis [3], [4], with good sensitivity and specificity, which consider clinical parameters like those considered in a classical way for diagnosis such as mental disorders, hypothermia, a precipitating factor and low levels of T45.

The pathophysiological substrate of MC lies in the intracellular decrease of T3 secondary to HT, which causes hypothermia and cardiac depression. Compensating mechanisms such as chronic peripheral vasoconstriction, moderate diastolic hypertension, and decreased blood volume are triggered. At this point, the presence of a trigger would end up breaking this fragile equilibrium [1].

\section{ETIOLOGY}

Up to $95 \%$ of MC cases occur in patients with primary HT [6]. In most of the cases reported, MC occurs in patients with HT after abandonment of treatment, as well as in those

\section{Published on June 26, 2020.}

T. Abderrahim, CHU Mohammed-VI Oujda, Morocco.

(corresponding e-mail: talhabderrahim ${ }^{@}$ gmail.com).

B. Houssam, CHU Mohammed-VI Oujda, Morocco.

H. Brahim, CHU Mohammed-VI Oujda, Morocco. under regular treatment in whom some physiological stress situation would trigger the event. Less frequently, cases of MC have been described in patients who are debutants with HT, in which the multiple clinical manifestations can represent a true diagnostic challenge.

TABLE I: MC TRIGGERING FACTORS

\begin{tabular}{|l|}
\hline HT debut \\
\hline Suspension of treatment \\
Cold exposure \\
Decompensated Congestive Cardiac failure, \\
Acute myocardial infarction \\
Burns or trauma \\
Stroke \\
Sepsis or infections \\
Metabolic \\
Hypoglycemia, Hyponatremia, Hypercapnia) \\
Medications \\
Gastrointestinal bleeding \\
Anesthetic procedures \\
\hline Surgical procedures \\
Labor \\
Ingestion of raw bok choy \\
\hline
\end{tabular}

There are multiple possible triggers of MC mostly correspond to situations of physiological stress. They have been described from cold exposure, stroke, heart failure, acute myocardial infarction, infectious processes and sepsis, trauma or fractures and electrolyte imbalances [5], [6]. Decompensations of diabetes mellitus such as diabetic ketoacidosis or hyperglycemic hyperosmolar state are less frequent. The use of different drugs (See Table I), especially amiodarone [7], and those used in anesthetic procedures and surgical events [8] are another trigger factors. There are some reports of labor as a trigger [9]. In a trivial way, the consumption of raw bok choy, a food rich in glucosinolates, whose metabolism generates products with inhibitory activity on the uptake of iodine by the Thyroid gland10 has also been described.

\section{ClinicAl MANIFESTATIONS}

\section{A. Hypothermia}

Caused by the decrease in thermogenesis, resulting from hypo metabolism, occurs in most cases, and can go beyond $24{ }^{\circ} \mathrm{C}$. It has been considered as a prognostic marker, since body temperature is equal to or less than $32{ }^{\circ} \mathrm{C}$, it is associated with higher mortality, which is why in some 
patients an extracorporeal circulation rewarming has been and normal temperature, an infectious process should be suspected. Used with good results [10] Hypothermia is so characteristic, that, in patients with compatible clinical symptoms of MC When you submit your final version, after your paper has been accepted, prepare it in two-column format, including figures and tables.

\section{B. Neurological alterations}

Historically, diagnosis was only considered in comatose patients. Today, the spectrum of neurological manifestations from a state of confusion, lethargy, obtundation, and even present seizures and status epilepticus. The etiology of these alterations is considered multifactorial: hypoglycemia, hyponatremia or hypoxemia due to cerebral low perfusion [11]. Anecdotally, even an entity called myxedematous psychosis has been described, characterized by behavioral alterations, aggressiveness, delusional ideation of damage and control [12], [13].

\section{Ventilatory alterations}

Caused by a poor response to hypoxia and hypercapnia, weaknesses of the respiratory muscles due to myopathy, obstructive sleep apnea and mechanical obstruction due to macroglossia6. They present alveolar hypoventilation, hypoxemia and hypercapnia, including requiring mechanical ventilation for prolonged periods [14] - [16].

\section{Cardiovascular alterations}

Considered the main cause of mortality and a common denominator in the patient with MC which is why it is the most extended part of the spectrum studied. Bradycardia and hypotension decreased myocardial contractility and cardiac output. There is a narrowing of the pulse, with elevation of the systolic pressure [11], [17]. Cases of congestive heart failure have been reported; however, because there is a decrease in oxygen demands [18], [19]. It is rare in the absence of persistent heart disease. Pericardial effusion is frequent and can be of large volume without necessarily representing the hemodynamics given the chronicity of its evolution.

An entity known as "heart myxedema" is described, represented by hemodynamic alterations, cardiomegaly, electrocardiographic alterations, and enzymatic alterations (increase in CPK, LDH and AST). In general, the "heart of myxedema" has a reversible behavior with the specific treatment [11]. The management of these manifestations focuses on hormonal supplementation, in some cases requiring inotropic and aminergic support.

\section{E. Gastrointestinal alterations}

There is a decrease in peristalsis; constipation is very frequent [13], which can even manifest as myxedematous megacolon. Myxedematous ileus may also occur, which is a gaseous distension of the abdomen accompanied by a clinical obstructive abdominal syndrome [14].

\section{F. Renal alterations}

It is the most frequent finding in patients with $\mathrm{MC}$, present in $43 \%$ of cases [3]. In general, of multifactorial origin, due to a fall in cardiac output and hypoperfusion, due to a decrease in glomerular filtration or a consequence of acute urinary retention, etc. The presentation of acute kidney injury caused by rhabdomyolysis secondary to hypothyroid myopathy has been described [25], [26].

\section{G. Hyponatremia}

Frequent electrolyte alteration, secondary of multiple mechanisms. One of them is the hyperproduction of $\mathrm{ADH}$ due to excess TSH with the consequent water retention [15] although today it has been questioned [24]. It may also be due to concomitant adrenal insufficiency, to acute renal injury or to being multifactorial. If it is severe it can contribute to neurological deterioration, and if it is mild, it has no clinical significance.

\section{H. Hypoglycemia}

It is present in $29 \%$ of cases [3]. It may be secondary to hypothyroidism itself due to a decrease in gluconeogenesis, a decrease in skeletal muscle glucose and adipose tissue [20] - [23]; or, it may be due to fasting and gastric paresis. It has been considered secondary to concomitant adrenal insufficiency or panhypopituitarism. In patients with HT, there is an alteration of plasma cortisol response to insulininduced hypoglycemia. It has also been postulated that adrenal insufficiency is precipitated by stress or by rapidonset replacement therapy [22]. Recently, it has been documented that women with chronic autoimmune hypothyroidism, the RR of suffering from Adisson's disease is 130 [24].

\section{Myxedema}

Described as hard, generalized edema, which does not leave a fovea, and includes the peri-orbital and acral area. There is supraciliary madarosis, known as "Queen Anne's sign" [25]. The skin is cold and dry due to reflected cutaneous vasoconstriction, pale yellowish due to anemia and hypercarotenemia, and rough to the touch [26], [27].

\section{DIAGNOSIS}

It is based on the clinical suspicion of a patient with or without a history of hypothyroidism, compatible clinical findings and the presence of precipitating factors.

Currently tools for diagnosis have been developed, which consider the thyroid profile and clinical parameters [3], [4]. The "diagnostic scoring system for myxedema coma" [3], developed in 2014, has been validated with better results; includes only clinical parameters, scoring each one. Take as a cut 60 points to consider the diagnosis of $\mathrm{MC}$, with sensitivity of $100 \%$ and specificity of $85 \%$ (See Table II). Higher scores increase specificity. This score has the advantage of being based solely on clinical parameters, therefore, the diagnosis will not be delayed until laboratory results are obtained and with this, early treatment could be initiated. Other screening tool, published in 2015 [4], includes, in addition to clinical parameters, measurements of $\mathrm{TSH}$ and F T-4, classifies the diagnostic probability and issues a therapeutic recommendation according to the score (See Table III); however, it has lower sensitivity and specificity $(80-80 \%)$. 
TABLE II: DIAGNOSTIC SCORING SYSTEM FOR MYXEDEMA COMA

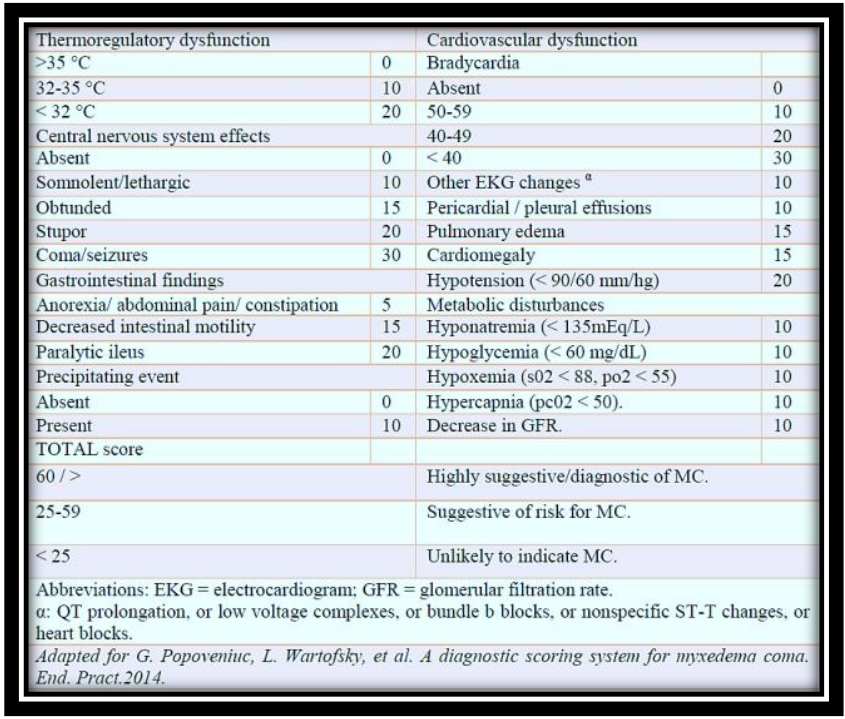

TABLE III: DIAGNOSTIC SCORING SYSTEM FOR MYXEDEMA COMA

\begin{tabular}{|c|c|c|}
\hline \multicolumn{3}{|c|}{$\begin{array}{l}\text { CRITERION } \\
\text { Score }\end{array}$} \\
\hline \multicolumn{3}{|c|}{ Glasgow CS } \\
\hline \multicolumn{2}{|c|}{$0-10$} & 4 \\
\hline \multicolumn{2}{|l|}{$11-13$} & 3 \\
\hline \multicolumn{2}{|l|}{14} & 2 \\
\hline \multicolumn{2}{|l|}{15} & 0 \\
\hline \multicolumn{3}{|l|}{ TSH } \\
\hline \multicolumn{2}{|l|}{$>30 \mathrm{Mu} / \mathrm{L}$} & 2 \\
\hline \multicolumn{2}{|l|}{$15-30$} & 1 \\
\hline \multicolumn{2}{|c|}{ Low T4 L $(<0.6 \mathrm{ng} / \mathrm{dL})$} & 1 \\
\hline \multicolumn{2}{|c|}{ Hypothermia $\left(<95^{\circ} \mathrm{F}\right)$} & 1 \\
\hline \multicolumn{2}{|c|}{ Bradycardia $(<60)$} & 1 \\
\hline \multicolumn{2}{|c|}{ Precipitating event } & 1 \\
\hline \multirow{3}{*}{$\begin{array}{l}\text { Total score } \\
8-10 \\
5-7\end{array}$} & Category & Recommendation \\
\hline & Most likely & Treat \\
\hline & Likely & $\begin{array}{l}\text { Treat if there are } \\
\text { no other causes }\end{array}$ \\
\hline & Unlikely & $\begin{array}{l}\text { Consider other } \\
\text { diagnosis }\end{array}$ \\
\hline \multicolumn{3}{|c|}{$\begin{array}{l}\text { Adapted for Chiong, et al. Development of an } \\
\text { objective tool for the diagnosis of myxedema coma. } \\
2015^{4} \text {. }\end{array}$} \\
\hline
\end{tabular}

Frequent electrolyte alteration, secondary of multiple mechanisms. One of them is the hyperproduction of $\mathrm{ADH}$ due to excess TSH with the consequent water retention [15] although today it has been questioned [24]. It may also be due to concomitant adrenal insufficiency, to acute renal injury or to being multifactorial. If it is severe it can contribute to neurological deterioration, and if it is mild, it has no clinical significance.

It is rare in the absence of persistent heart disease. Pericardial effusion is frequent and can be of large volume without necessarily representing the hemodynamics given the chronicity of its evolution.

\section{TREATMENT}

The management of the patient with $\mathrm{MC}$ is a real medical emergency, since the punctual onset of it implies an improvement in the prognosis, delaying diagnosis will increase mortality, so that having a justified clinical suspicion; it should be started as early as possible without waiting the lab results.

The goals regarding MC management should be aimed at improving neurological, cardiovascular and pulmonary manifestations [27] - [31]. Management in intensive care should be provided and remain under monitoring of the cardiorespiratory status, receive the pertinent ventilatory assistance, effective water resuscitation and correction of hypotension and electrolyte alterations. It is recommended to initiate passive rewarming, since active rewarming could generate peripheral vasodilatation, generating cardiovascular collapse [32]. The identified trigger must be investigated and treated [33].

Regarding hormonal supplementation, the current recommendations are based on reports of case series and expert opinions, since due to the low frequency of MC it is not feasible to design appropriate clinical trials.

L-thyroxine, the first synthetic molecule used for the management of hypothyroidism [34], remains the mainstay of treatment. It is suggested to start with initial loading dose of 300-400 mcg, always if possible intravenous (IV). Subsequent supplementation should be continued with $1.6 \mathrm{mcg} / \mathrm{kg}$, or $75 \%$ of this dose in case of IV administration [31], this being the ideal route, due to the best clinical and paraclinical results shown in the short term [33]. The enteral route is less preferred due to the alterations in intestinal absorption that these patients have, as well as the need for immediate bioavailability of thyroid hormone [34] - [36].

Taking into account that, in most of cases, MC patients present with peripheral conversion reduction, the adjunctive therapy with L-T4 + L-T3, already recommended in some specific groups of patients with HT [28], [29], should also be used in MC. It is also preferred to use it intravenously and administer it at low doses, since it has been documented that doses equal to or greater than $75 \mathrm{mcg} /$ day are associated with higher mortality [32]. An initial dose of 5-20 mcg is recommended, followed by fractionated doses, at a rate of $2.5-10 \mathrm{mcg}, 3$ times a day, continuing at least until significant clinical improvement is obtained.

Glucocorticoid supplementation is indicated empirically in all cases at a dose of stress, before starting supplementation with levothyroxine, and having previously requested plasma levels of cortisol [31]. It is recommended to initiate hydrocortisone $100 \mathrm{mg}$ intravenously, followed by $50 \mathrm{mg}$ intravenously every 6 hours. It should be continued until concomitant adrenal insufficiency is ruled out.

\section{PROGNOSIS}

Today, MC mortality remains high, although it has changed due to better knowledge of the pathology, the development of instruments to facilitate faster recognition and a more intensive therapy. In the $60 \mathrm{~s}$, mortality was up to $80 \%$ [35]. With the beginning of the use of intravenous L$\mathrm{T} 4$, in the 1990s it decreased significantly, reporting figures of $20-25 \%$ [36], identifying cardiovascular failure as the main cause, followed by pneumonia and its complications [32]. A retrospective observational study, which included 
149 patients, published in 2017, showed that there have been few changes regarding mortality, placing it at around $29 \%$ [37], and finding cardiovascular failure as the main cause.

\section{CONCLUSION}

The prognosis is becoming better with this emergency. It has been identified that the best predictors of poor prognosis are the deterioration of the level of consciousness, Glasgow scale equal to or less than 5, and a score equal to or greater than 20 for APACHE II. More recently, it was shown that the SOFA score is more effective than other models, so a score of 6 points at the beginning and at 3 days is highly predictive, with mortality greater than $60 \%$ from this cut-off point, which increases by increasing the score.

\section{REFERENCES}

[1] V. Mathew, R.A. Misgar, S. Ghosh, et al. Myxedema coma: a new look in to an old crisis. J Thyroid Res. 2011; 1: 493-462.

[2] J. Hampton. Thyroid gland disorder emergencies: thyroid storm and myxedema coma. AACN. Adv Crit Care. 2013; 24:325-32.

[3] G. Popoveniuc, L. Wartofsky, et al. A diagnostic scoring system for myxedema coma. Endocrine Practice, 2014; 20: 808-817.

[4] Yien V. Chiong. Development of an objective tool for the diagnosis of myxedema coma. Translational Research, 2015, 166(3): 233-245.

[5] J. Nicoloff, J. Lopresti. Myxedema coma, a form of decompensated hypothyroidism. Endocrinol Metab Clin North Am. 1993; 22: 279-90.

[6] L. Wartofsky, Myxedema Coma. Endocrinol Metab Clinic North America. 2006. 35: 687-698.

[7] S. Chakraborty, J. Fedderson, J.J. Gums , Toole A. Amiodaroneinduced myxedema coma: a case and review of the literature. Arch Med Sci. 2014; 6: 1263-1268.

[8] N. Stathatos, L. Wartofsky. Perioperative management of patients with hypothyroidism. Endocrinol Metab Clin North Am. 2003; 32:503-518.

[9] N.O. Turhan, M.C. Kockar, I. Inegol. Myxedematous coma in a laboring woman suggested a pre-eclamptic coma: a case report. Acta Obstet Gynecol Scand. 2004; 83:1089-1891.

[10] M. Chu, T.F. Seltzer. Myxedema coma induced by ingestion of raw bok choy, N England J Medicine. 2010. 362(20): 1945-1946.

[11] S. Danzi, I. Klein. Thyroid hormone and the cardiovascular system. Med Clin North Am. 2012; 96:257-268.

[12] I. Khochtali, N. Hamza . Reversible dilated cardiomyopathy caused by hypothyroidism. International Archives of Medicine. 2011; 4:2026.

[13] R.E Watanakunakorn, R.E Hodges. Myxedema: A Story of 400 Cases. Arch Intern Med. 1965; 116:183-190.

[14] J.C. Ravi Shankar, H. Ramesh, N. Hebsur. Hypothyroidism as a Rare Cause for Ogilvie Syndrome. Journal of Dental and Medical Sciences. 2015. 14(4): 32-34

[15] R.W Skowsky, T.A Kikuchi. The role of vasopressin in the impaired water excretion of myxedema. Am J Med 1978; 64: 613-621.

[16] V. Sanders. Neurologic manifestations of myxedema. New Engl Journal Medicine. 1962; 266:547-551.

[17] T.W Heinrich, G. Grahm. Hypothyroidism Presenting as Psychosis: Myxedema Madness Revisited. Prim Care Companion J Clin Psychiatry. 2003; 5:260-266.

[18] I. Espinoza, R. Ramos, A.M Maroon. Myxedema madness. Hipotiroidismo que debuta como psicosis. Rev Psiquiatr Salud Ment. 2010; 3(3): 111-112.

[19] W. Reinhardt, K. Mann. Incidence, clinical picture, and treatment of hypothyroid coma: results of a survey. Med Klin 1997; 92: 521-4.

[20] A. Kogan, K. Yigal. Severe hypothermia in myxedema coma: A rewarming by extracorporeal circulation. Emergency Medicine Australasia. 2011. 23: 773-775.

[21] T. Yamamoto. Delayed respiratory failure during the treatment of myxedema coma. Endocrinol Japan. 1984; 31: 769-775.

[22] T.C. Kamilaris, C.R DeBold, S.N. Pavlou. Effect of altered thyroid hormone levels on hypothalamic-pituitary-adrenal function. J Clin Endocrinol Metab. 1987; 65:994-999.

[23] S. Crunkhorn, M.E. Patti. Links between thyroid hormone action, oxidative metabolism, and diabetes risk? Thyroid. 2008; 18:227-237.
[24] Y. Iwasaki, Y. Oiso, K. Yamauchi. Osmoregulation of plasma vasopressin in myxedema. J Clin Endocrinol Metab. 1990; 70:534539.

[25] M. Altay, M. Duranay, M. Ceri. Rhabdomyolysis due to hypothyroidism. Nephrol Dialysis Transp.2004. 20(4): 847-848.

[26] Z.Y. Chang, A.Y. Boo. Rhabdomyolysis: A rare complication of hypothyroidism. Proceedings of Singapore Healthcare 2015. 24(3): 188-190.

[27] F. Leonard, I. Rizzo, L. Daniela. Coma mixedematoso. Rev Medicina. 2017; 77: 321-328.

[28] B. Nygaard. Effect of combination therapy with thyroxine (T4) and 3,5,3-triiodothyronine versus $\mathrm{T} 4$ monotherapy in patients with hypothyroidism, a double-blind, randomised cross-over study. Eur J Endocrinol 2009; 161: 895-902.

[29] E.A. McAninch, A.C. Bianco. New insights into the variable effectiveness of levothyroxine monotherapy for hypothyroidism. Lancet Diabetes Endocrinol. 2015; 3: 756-758.

[30] J. Garber, R. Cobin, H. Gharib. Clinical practice guidelines for hypothyroidism in adults: cosponsored by the American Association of Clinical Endocrinologists and the American Thyroid Association. Endocr Pract 2012; 18 (6):1004.

[31] J. Jonklaas, C.B. Antonio. Guidelines for the Treatment of Hypothyroidism. Am. Thyroid Assoc. Thyroid. 2014. 24(12): 16701752.

[32] T. Yamamoto, J. Fukuyama, A. Fujiyoshi. Factors associated with mortality of myxedema coma: report of eight cases and literature survey. Thyroid. 2009. 9: 1167-1174.

[33] P.W. Ladenson, P.D. Goldenheim, D.S. Cooper, M.A. Miller. Early peripheral responses to intravenous L-thyroxine in primary hypothyroidism. Am J Med. 1982. 73:467-474.

[34] K. Boelaert. Prevalence and Relative Risk of Other Autoimmune Diseases in Subjects with Autoimmune Thyroid Disease. American J Med. 2010. 123(12): 1831-9.

[35] J.F. Nickerson, S.R Hill, J.H. McNeil, S.B Barker. Fatal myxedema, with or without coma. Ann Intern Med. 1999. 53;175-193.

[36] R.M. Jordan. Myxedema coma. Pathophysiology, therapy, and factors affecting prognosis. Medical Clinics of North America. 1995. 79:185194.

[37] O. Yosuke, O. Sachiko. Clinical characteristics and outcomes of myxedema coma: Analysis of a national inpatient database in Japan. Journal of Epidemiology. 2017. 27: 117-122.

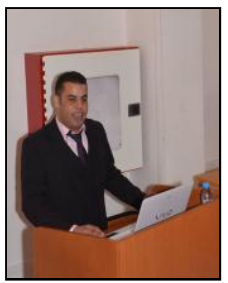

A. Talha is the first author. He is medical doctor and a specialist in intensive care and anesthesia at the hospital university center of Oujda in Morocco. He is known as an academic researcher and has too many conferences in several topics related with anesthesia and intensive care. 